Check for updates

Cite this: Phys. Chem. Chem. Phys., 2018, 20, 16719

Received 23rd March 2018,

Accepted 29th May 2018

DOI: $10.1039 / c 8 c p 01906 a$

rsc.li/pccp

\title{
Size dependent surface charge properties of silica nano-channels: double layer overlap and inlet/outlet effects $\dagger$
}

\author{
Tumcan Sen and Murat Barisik (D) *
}

\begin{abstract}
Transport inside nano-channels and tubes is highly dependent on their surface charge properties. While previous studies assume that the charge density of a surface is a material property and independent of confinement size, this study properly characterized the surface charge of a nanochannel as a function of channel height and length under various solution conditions. By calculating the local surface charge based on local ionic concentrations, the surface charge of a nano-channel was studied by considering the effects of both overlapping electrical double layers (EDLs) and inlet/outlet regions. First, the surface charge of silica decreased with the increase in EDL overlap, which is characterized by the ratio of EDL thickness to channel height. Second, the local surface charge showed variation at the inlet/outlet regions where the channel's electrokinetics was in development. We defined a general entrance length as a function of EDL thickness for the electrokinetically developing part of different cases, after which the surface charge reached its equilibrium value and remained constant. Based on such length scales, we extended the existing theory to include nano-effects. A phenomenological model was developed, which can predict the average nano-channel surface charge as a function of EDL thickness, $\mathrm{pH}$, channel length and channel height.
\end{abstract}

In recent years, nanoscale systems have become increasingly popular in various ranges of applications such as DNA analyzers, ${ }^{1-3}$ DNA and protein transport, ${ }^{4-8}$ drug delivery, ${ }^{9-12}$ biological/chemical agent detection, ${ }^{13,14}$ micro/nano chip sensors, ${ }^{15-17}$ and nanomembranes. ${ }^{13,18}$ In most of these systems, the charge property of solid surfaces in contact with the aqueous solution plays a key role. For example, force or electrokinetic-driven transport of ions, fluid, and particles within microfluidic and nanofluidic devices highly depends on the surface charge densities of the channel walls. Hence, the design and performance of these applications requires an accurate knowledge of the surface charge properties of nanoscale confinements in various ionic solutions, which is not yet well understood.

A surface in contact with an aqueous medium becomes charged due to dissociation of ionizable groups or adsorption/ reaction of ions at the interface. ${ }^{19,20}$ The resulting surface charge interacting with dissolved ions forms an electrical double layer (EDL) on the charged surface in which more counter ions accumulate and co-ions lessen. ${ }^{21}$ Transport inside micro/nano channels and tubes develops as a function of the wall charge properties. For example, the process of desalination through a

Department of Mechanical Engineering, Izmir Institute of Technology, IZMIR, 35430, Turkey. E-mail: muratbarisik@iyte.edu.tr

$\dagger$ Electronic supplementary information (ESI) available. See DOI: 10.1039/c8cp01906a nano-porous membrane depends on the surface charge density of the pore network. ${ }^{22}$ Many of the existing studies and applications assume that the charge density of a surface is a material property and independent of the confinement size. ${ }^{23-25}$ However, for small confinement heights comparable with the thickness of the EDL, ionic layers (EDLs) extending from opposite surfaces overlap, causing the local ionic concentrations inside the EDL and the surface charge to deviate from theoretical assumptions. ${ }^{26,27}$ Since surface reactions depend on the local ionic environment such as local $\mathrm{pH}$ and salt concentrations at the interface, the surface charge of an overlapped EDL becomes different from the surface charge calculated for the non-overlapping case.

In addition to the EDL overlap developing in the surfacenormal-direction due to small characteristic heights, local ionic concentration variations at the inlet and outlet of channels/ pores also become dominant in short length conduits of recent nanotechnological applications. For example, diffusion of ions from bigger voids into pores in a nano-membrane pore-network creates a different ionic equilibrium through the entrance and exit regions of every pore. As a result, variation of local solution properties on the surface in the axial direction creates variation of the surface charge in these entrance regions. Further complications develop in the case of flow conditions. For example, concentration polarization at the entrance region of membrane devices becomes dominant particularly for high degrees of EDL 
overlap and short length conduits, which substantially affects the filtration mechanisms. ${ }^{28,29}$ Transport of ions by a pressure gradient also promotes the axial variation of electrical potential, which opposes the motion of fluid in the axial direction. ${ }^{30}$ In order to distinguish these effects developing in fluid flows, the surface charge density and the resulting electric potential distribution within the short nano-channel at a stationary state should be resolved.

The calculation of surface charge density as a function of the local ionic environment has been practiced by utilizing some active surface charge models in the literature. The so-called "Charge Regulation (CR)" nature of surface chemistry was first identified by Ninham and Parsegian ${ }^{31}$ and later observed by multiple experiments in surface force measurements by colloids ${ }^{32-35}$ and AFM. ${ }^{36-40}$ Since then, researchers have attempted to implement CR into numerical calculations of ionic distributions in nanosystems using active charge models as a boundary condition. CR models calculate the effects of the protonation/deprotonation in surface reactions based on the site density of the functional groups. In recent years, CR models have received significant attention from various research fields from colloidal science to atomic force measurement techniques. ${ }^{41-45}$ In particular, studies dedicated to nano-tubes/channels generally focused on flow conditions to calculate the ionic conductance and streaming current. $^{46-48}$ Most of these existing studies focus on the surface charging characteristics of straight channels and tubes which are assumed to be infinite in length. ${ }^{49-53}$ However, the average surface charge of a finite-length conduit becomes different from the surface charge developing sufficiently away from inlets and outlets, depending on the channel length. There are only a few studies in the literature that refer to these effects ${ }^{54-56}$ but these studies presented their results under flow conditions as an observation at a specific channel height and length without examining or describing its occurrence ${ }^{57}$ and for long channels disregarding the end effects. ${ }^{58}$ Hence, there is no suitable characterization or explicit solution for the surface charge behavior of short nano-conduits, yet.

Our objective is to characterize the surface charge density of nanochannels of various heights and lengths under various solution conditions. We study silica surfaces theoretically since it has been widely used in recent applications. ${ }^{59-61}$ For the first time in the literature, this study performs characterization of the effects of both overlapping EDL fields and inlet/outlet regions for short nano-conduits with stationary fluid as a function of channel height, channel length and solution conditions; and provides an empirical model as an extension of existing theory. A multi-ion charge-regulation model ${ }^{61}$ is utilized, which associates the surface charging behavior to the ionic aqueous solution properties, mainly $\mathrm{pH}$ and concentration of the solution. The current model is validated by the cases with available analytical solutions.

\section{Theoretical background and mathematical model}

Generally, ionic distribution on a surface with a given surface charge density obeys the Boltzmann distribution (BD). ${ }^{62-64}$
Hence, the potential distribution within a semi-infinite EDL can be resolved by solving the well-known Poisson-Boltzmann (PB) equation. The PB equation can be easily solved analytically for low wall potentials by utilizing Debye-Hückel simplification. ${ }^{65}$ For high wall potential cases, some studies solve very complex elliptic functions due to nonlinearity. ${ }^{66-68}$ If the EDLs growing from opposite parallel surfaces start interacting (i.e. EDL overlapping), implementing the Boltzmann distribution to the Poisson equation becomes incorrect. ${ }^{49,69}$ This fact can easily be seen in Fig. 1, which shows the overlapped and non-overlapped electric potential distributions within a channel in the case of different scenarios. To begin, the potential distribution with EDL overlap significantly deviates from the no-overlap case that obeys the Boltzmann distribution. For such a case, the original form of the PoissonNernst-Planck (PNP) theory should be employed in the analyses, in contrast to frequently used simplified versions of PNP. ${ }^{62,69}$

Another significant point to mention in Fig. 1 is the current perspective about the surface boundary condition definitions of the existing studies solving the potential distribution of overlapped EDLs. In the literature, assigning either constant surface charge $^{70-72}$ or constant wall potential ${ }^{30,73,74}$ boundary conditions onto the surfaces is the general way to solve PNP. Eqn (1) describes the relation between the wall potential $(\psi)$ and surface charge density on the wall $\left(\sigma_{\mathrm{w}}\right)$. If constant surface charge density is assumed on both surfaces (Case 1 in Fig. 1), the electric potential value at the surface will change by EDL overlap. On the other hand, if a constant wall potential condition is implied (Case 2 in Fig. 1), EDL overlap will cause the change of the surface charge density (i.e. slope of the potential distribution at the wall). These cases are common practices in existing literature, however, both approaches do not properly reproduce the nature of the surface.

$$
-\varepsilon_{0} \varepsilon_{\mathrm{r}} \vec{n} \cdot \nabla \psi=\sigma_{\mathrm{w}}
$$

Enforcing constant surface charge or potential at the interface does not represent the physical response of the surface chemistry. The surface reactions develop based on the local ionic environment at the interface, that neither surface charge nor potential remain constant; instead, both of them undergo variation in response to the variation in ionic distribution and create a new equilibrium, accordingly. Such behavior is

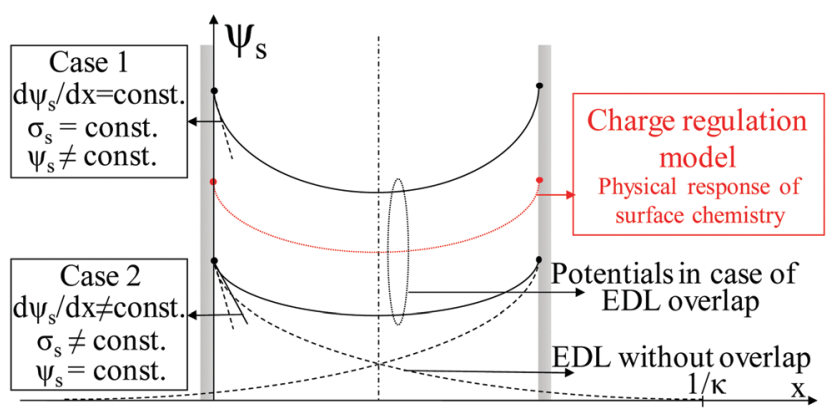

Fig. 1 Distribution of electric potential with and without EDL overlap. In the case of EDL overlap, two generally employed boundary conditions of constant surface charge (Case 1 ) and constant surface potential (Case 2 ) in comparison with the charge regulation model used in this study. 
modelled by the previously developed multi-ion charge-regulation model, which considers the effects of the protonation/deprotonation surface reactions, the site density of the functional groups, and the $\mathrm{pH}$ and salt concentration of the aqueous solution on the silica surface. This boundary condition model forms the basis of the current study.

In the current model, a slit like silica nano channel, which is connected to reservoirs at both ends, is considered as illustrated in Fig. $\mathrm{S} 1$ (ESI $\dagger$ ). The liquid phase is a $\mathrm{KCl}$ (i.e. symmetric $1: 1$ ) aqueous electrolyte solution consisting of 4 types of ionic species namely; $\mathrm{H}^{+}, \mathrm{K}^{+}, \mathrm{Cl}^{-}$and $\mathrm{OH}^{-}$ions with their bulk values being $c_{10}, c_{20}, c_{30}$ and $c_{40}$, respectively. Due to protonation/ deprotonation of ions, the silica surface is charged when in contact with an ionic solution. The surface charge is mainly controlled by the $\mathrm{K}^{+}$and $\mathrm{Cl}^{-}$ions while the $\mathrm{pH}$ of the solution is adjusted by the $\mathrm{H}^{+}$and $\mathrm{OH}^{-}$ions. Detailed information on the Poisson-Nernst-Plank (PNP) equations employed in the study is given in the ESI. $\dagger$

As a result of protonation/deprotonation reactions taking place at the solid/liquid interface, the silica channel walls show a charge-regulated behavior depending on the ionic concentration and $\mathrm{pH}$ of the ionic aqueous solution. In order to implement this charge regulated nature into the model, two fundamental dissociation/association reactions are considered at the solid/ liquid interface as:

$$
\begin{gathered}
\mathrm{SiOH} \leftrightarrow \mathrm{SiO}^{-}+\mathrm{H}^{+} \\
\mathrm{SiOH}+\mathrm{H}^{+} \leftrightarrow \mathrm{SiOH}_{2}^{+}
\end{gathered}
$$

The equilibrium constants are evaluated by using

$$
K_{\mathrm{A}}=\frac{\Gamma_{\mathrm{SiO}^{-}}\left[\mathrm{H}^{+}\right]_{\mathrm{w}}}{\Gamma_{\mathrm{SiOH}}}, \quad K_{\mathrm{B}}=\frac{\Gamma_{\mathrm{SiOH}_{2}^{+}}}{\Gamma_{\mathrm{SiOH}}\left[\mathrm{H}^{+}\right]_{\mathrm{w}}},
$$

where $\Gamma_{\mathrm{SiO}^{-}}, \Gamma_{\mathrm{SiOH}}$ and $\Gamma_{\mathrm{SiOH}_{2}}{ }^{+}$are the surface site densities of $\mathrm{SiO}^{-}, \mathrm{SiOH}$ and $\mathrm{SiOH}_{2}{ }^{+}$, respectively and $\left[\mathrm{H}^{+}\right]_{\mathrm{w}}$ is the hydrogen concentration at the solid/liquid interface. In this regard, the surface charge density of the silica walls can be denoted as:

$$
\sigma_{\mathrm{w}}=-\frac{F \Gamma_{\text {total }}}{N_{\mathrm{A}}} \frac{K_{\mathrm{A}}-K_{\mathrm{B}}\left[\mathrm{H}^{+}\right]_{\mathrm{w}}{ }^{2}}{K_{\mathrm{A}}+\left[\mathrm{H}^{+}\right]_{\mathrm{w}}+K_{\mathrm{B}}\left[\mathrm{H}^{+}\right]_{\mathrm{w}}{ }^{2}}
$$

\section{Results and discussion}

We initiated our study by calculating the ionic distribution inside a $10 \mathrm{~nm}$ height and $30 \mathrm{~nm}$ length channel. The electric potential contours of $C_{\mathrm{KCl}}=1 \mathrm{mM}, 10 \mathrm{mM}$ and $100 \mathrm{mM}$ cases, which define the $\lambda / H$ ratio, are given at $\mathrm{pH}=4,6$, and 8 in Fig. 2 . Here, Debye length $(\lambda)$ is normalized with the half channel height $(H)$ so that the degree of EDL overlap within the channel can be characterized by the non-dimensional number, $\lambda / H$. Local electric potential shows variation inside the channel as well as through the reservoir region near to the channel inlet and outlet as a function of ionic concentration and $\mathrm{pH}$. Simply, the ionic distribution along the channel is spatially uniform and the electrical potential at the channel center away from the surfaces is equal to the bulk reservoir value, when the channel

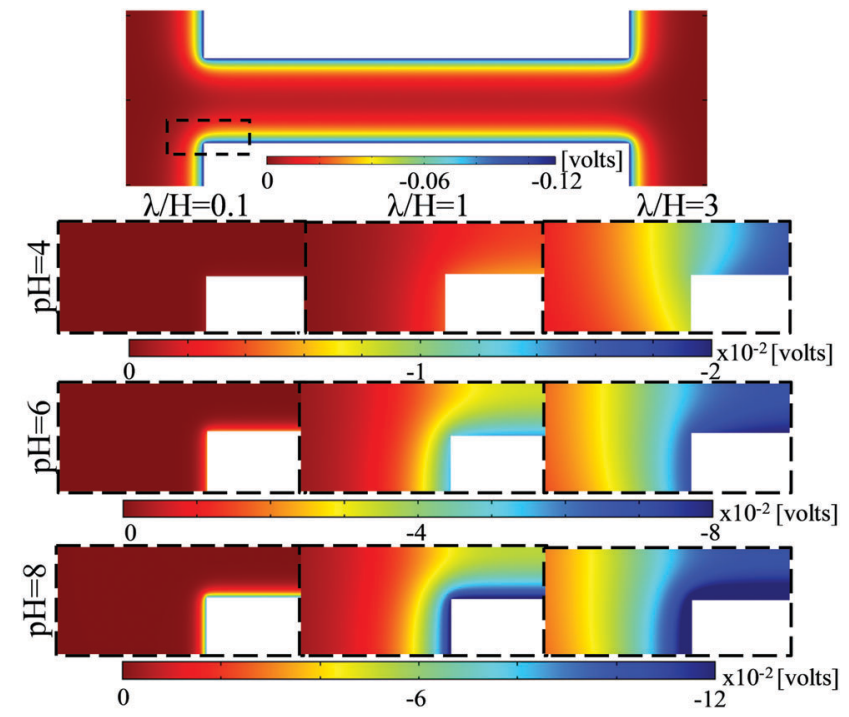

Fig. 2 Electric potential distribution for different $\mathrm{pH}$ and overlapping ratios.

height is much larger than the EDL thickness (i.e., $\lambda / H \ll 1$ ). However, as the EDL thickness grows, EDL overlap starts and the electrical potential in the channel becomes different from the reservoirs. At this point, we specified these ionic variations based on their physical origins. First, overlapping EDLs from top and bottom surfaces affect electric potential inside the channel. Second, diffusion from the reservoir creates electric potential variation near the inlet/outlet regions. The latter effect also includes curvature effects developing at the corners of the channel ends, which will be discussed in detail later.

Next, we calculated local surface charge densities based on the ionic potential formed on the surface. Fig. 3 gives the distribution of surface charge along half of the $30 \mathrm{~nm}$-length channel as a function of $\mathrm{pH}$ and overlap ratio. Different surface charge values develop as a result of different bulk ion concentrations at each $\lambda / H$ case. The first observation from Fig. 3 is the surface charge behavior along the channel. At low $\lambda$ values creating low $\lambda / H$ cases, charge is mostly constant on the channel surface except the very early inlet region. However, an increase of $\lambda$ yields further penetration of this entrance effect into the channel such that the surface charge cannot reach an equilibrium value even up to the middle of the channel for cases $\lambda / H=1$ and 3. Overall, local variation through the channel increases with the increase of EDL thickness and a "developing region" forms at both ends of the nano-channel in which the surface charge density shows unusual behavior.

In order to characterize this developing region, we performed calculations further for different channel lengths. We measured the local surface charges at the middle of each channel and normalized them with theoretical calculations of surface charge density at the corresponding salt concentration and $\mathrm{pH}$ values. The results are summarized in Fig. S3 (ESI $\dagger$ ). Flat surface theory can successfully calculate the nanochannel surface charge at mid-length for low overlap ratios $(i . e . \lambda / H)$ such as 0.1 and 0.3 . However, the nanochannel surface charge density significantly 

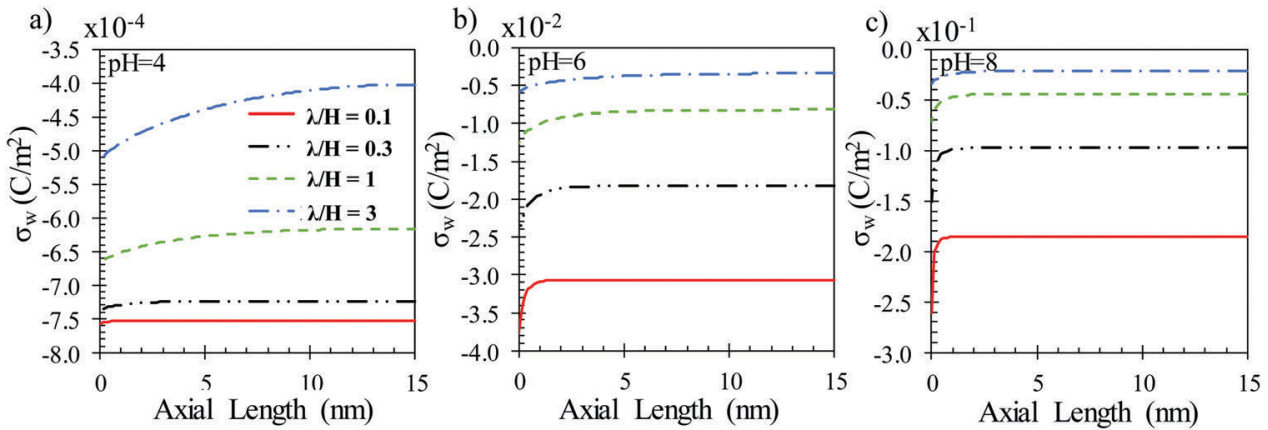

Fig. 3 Surface charge density distribution in a $30 \mathrm{~nm}$ long channel with $10 \mathrm{~nm}$ height of the silica wall at different EDL overlaps for (a) $\mathrm{pH}=4$, (b) $\mathrm{pH}=6$, and $(\mathrm{c}) \mathrm{pH}=8$.

differs from theoretical predictions as the EDL overlap develops. An increase in overlap ratio yields lower surface charges than the theoretical estimations. Moreover, entrance region effects are dominant at high $\lambda$ cases where local surface charge along the nanochannel continues to vary and cannot reach its equilibrium value up to $50-100 \mathrm{~nm}$ channel lengths depending on the $\mathrm{pH}$. It can be concluded that the developing region effects are more significant as $\mathrm{pH}$ decreases and EDL thickness increases.

Based on the results from Fig. S3 (ESI $\dagger$ ), we proceeded with our investigations by using longer length channels to ensure that an ionic equilibrium was formed at least in the mid-length of the channel. Local surface charge values through the channel wall normalized by the equilibrium charge value $\left(\sigma_{\text {bulk }}\right)$ of the corresponding channel are presented in Fig. 4. This time, we also normalized the axial channel direction with EDL thickness to develop a possible characterization for the length scale of the developing region. With the exception of those with a low $\mathrm{pH}$ value of 4 , cases with different overlap ratios showed very similar charge distributions in the developing region. Through this distribution, the developing region yielded significantly higher surface charge values, which has a substantial influence on the average surface charge of a nanochannel. For a general description, we defined the "developing length" for surface electrokinetics $\left(L_{\mathrm{e}}\right)$ as the axial position where the surface charge density reaches $99 \%$ of the equilibrium value developing away from the inlets/outlets. Normalized developing lengths estimated for different $\lambda / H$ cases are given in Fig. S4 (ESI $\dagger$ ) for different $\mathrm{pH}$ values. As the $L_{\mathrm{e}}$ is normalized by the EDL thickness, Fig. S4 (ESI $\dagger$ ) shows purely the effect of EDL overlap, independent of the effect of EDL thickness on the developing region. Overall, $L_{\mathrm{e}}$ shows a decreasing trend by the increase of EDL overlap, and an increasing trend by the decrease in $\mathrm{pH}$. The longest $L_{\mathrm{e}}$ value is measured as $2.7 \times \lambda$.

In an attempt to develop a simple model to predict the average surface charge density of a given nanochannel, we tried to formulate deviations from theoretical predictions as a function of channel height, channel length, ionic salt concentration and $\mathrm{pH}$. By accomplishing this, we will be able to extend the existing theory to consider EDL overlap and developing region effects. For such purposes, we performed modelling of surface charge behavior in two regions; the developing region and developed region. First, we attempted to determine an $L_{\mathrm{e}}$ value to define an average surface charge value in the developing region. $L_{\mathrm{e}}$ gets different values ranging from $0.5 \times \lambda$ to $2.7 \times \lambda$, and variation is hard to formulate. For such a case, we decided to use the maximum value of $L_{\mathrm{e}}$ as the developing length for all cases $\left(L_{\mathrm{e}}=2.7 \times \lambda\right)$, to ensure that all local variations in every case are considered. Consequently, the average surface charge behavior before and after $2.7 \times \lambda$ (along axial direction) is expressed using two different models.

For the developing region, we observed two mechanisms creating the charging behavior. The first mechanism is the corner effect developing at the corners of the channel ends due to curvature. This effect was observed by multiple researchers
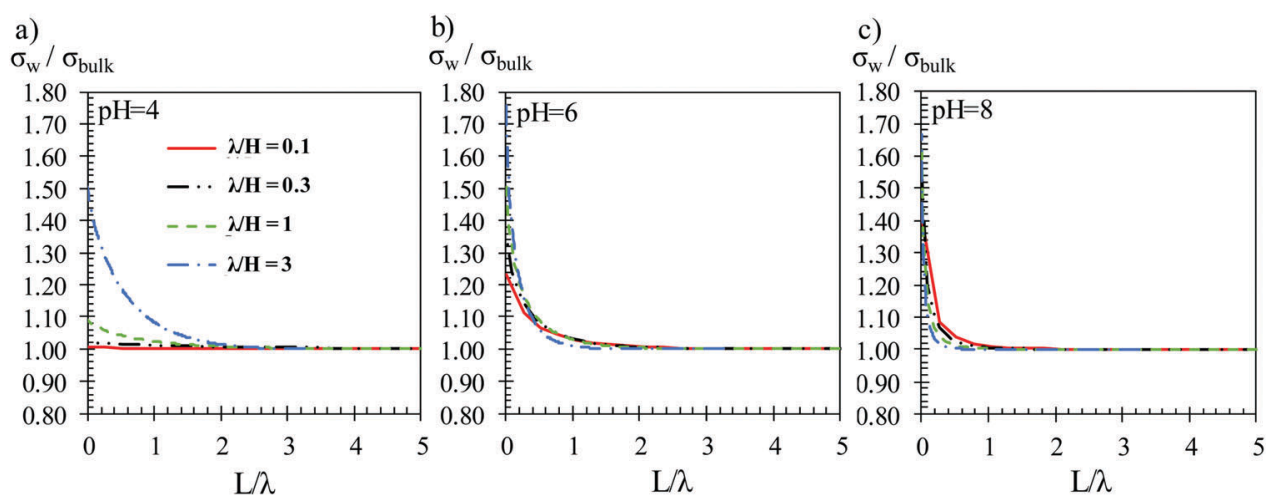

Fig. 4 Normalized charge density along the channel length normalized with the Debye length $(\lambda)$. 
in the surface charge behavior of nanoparticles when the surface to volume ratio decreased to a certain value. ${ }^{55,75}$ A very similar curvature effect is also observed on the capacitance behavior of mesoporous supercapacitors, ${ }^{76-78}$ in which the ion distributions significantly deviate from planar surface calculations by the decrease in the pore size. The second mechanism in the developing region is the interaction of ionic concentration developed in the channel with the reservoir. This effect only develops in the case of EDL overlap when the channel center ion distribution becomes different from the reservoir value. Our objective here is to distinguish the effect of these two mechanisms in developing region behavior.

First, we studied the local surface charge distributions forming on a simple corner geometry by itself in order to calculate the corner effects only at different concentrations and $\mathrm{pH}$ values. Fig. 5(a) shows the electric potential contour around a corner and illustrates the curvature effects. The distributions of surface charge density normalized by theoretical estimations along the axial direction normalized by the corresponding EDL thickness are given in Fig. 5(b)-(d) under different ionic conditions. Surface charge increased by moving towards the edge of the corner reaching almost twice the theoretical predictions. Similar behavior was observed in nano-particles where the decrease of particle size increased the surface charge value. ${ }^{61}$ Surface charge reached the theoretically calculated value sufficiently away from the corner, as expected. Variation of surface charge was mostly confined in the $L / \lambda \lesssim 2$ region. We further calculated a length scale for the corner effect by measuring the distance at which the surface charge density reaches $99 \%$ of the equilibrium value predicted by theory. The axial length of the corner effect was found to be similar to $L_{\mathrm{e}}$ measured for the developing region length earlier. Hence, we employed the $L_{\mathrm{e}}=2.7 \times \lambda$ value to characterize the corner effects.

Next, we excluded the corner effects from the behavior of a developing region by simply removing the variation of surface charge in Fig. 5(b)-(d) from the distributions of surface charge measured in the developing part of the channels presented in Fig. 3(a)-(c). The remaining parts presented in Fig. 5(e)-(g) show the effects of the second mechanism; interaction of ionic concentration developed in the channel with the reservoir only. For instance, in this $10 \mathrm{~nm}$ total channel height, $\lambda=0.5 \mathrm{~nm}$ results in $\lambda / H=0.1$ which means no EDL overlap, therefore only a corner effect develops and after removing it (Fig. 5(b)) from Fig. 3(a), the variation disappeared and the surface charge became equal to theory through all channels as given in Fig. 5(e). On the other hand, $\lambda=15 \mathrm{~nm}$ results in $\lambda / H=3$ showed strong EDL overlap where the equilibrium mid-length surface charge becomes lower than theory and there was slight variation through developing the length even though corner

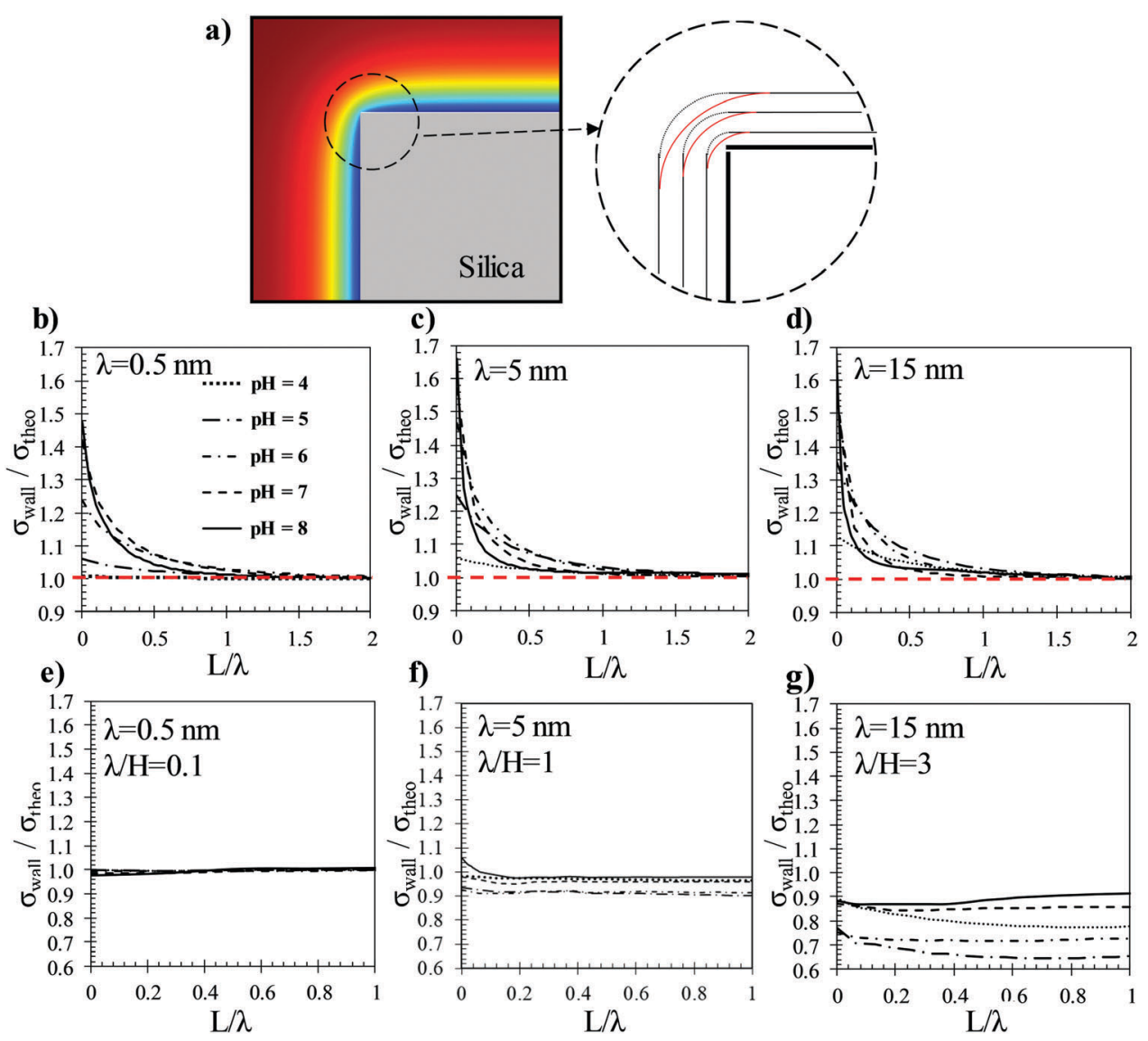

Fig. 5 (a) Variation of local ionic concentration at a corner and illustration of curvature effects. Normalized surface charge distributions (b)-(d) on a corner at different EDL thicknesses and $\mathrm{pHs}$, and $(\mathrm{e})-(\mathrm{g})$ in the developing region of a $10 \mathrm{~nm}$ height channel after the corresponding corner effects are removed. 
effects were removed. As explained, this behavior is due to the interaction of the reservoir with the new ionic equilibrium developed in the channel which is different from the reservoir value as the result of EDL overlap.

At this stage, we defined an average surface charge developed under each mechanism inside their corresponding length extents. We calculated an average charge through 0 to $2.7 \times \lambda$ length on the corner ( $\left.\sigma_{\text {corner-ave }}\right)$. Similarly, an average charge through 0 to $2.7 \times \lambda$ length was calculated inside the developing region $\left(\sigma_{\text {developing-ave }}\right)$. Finally, an average charge for the rest of the channel was defined as the developed section ( $\left.\sigma_{\text {developed }}\right)$. Basically, we integrated the surface charge variation on a corner and in the electrokinetically developing part of a channel by eqn (6) and (7), while the remaining part denoted by eqn (8) resulted in a constant value along the rest of the channel.

$$
\begin{gathered}
\sigma_{\text {corner-ave }}=\frac{1}{2.7 \times \lambda} \int_{0}^{2.7 \times \lambda} \sigma_{\text {corner }} \mathrm{d} x \\
\sigma_{\text {developing-ave }}=\frac{1}{2.7 \times \lambda} \int_{0}^{2.7 \times \lambda} \sigma_{\text {developing }} \mathrm{d} x \\
\sigma_{\text {developed-ave }}=\frac{1}{L / 2-2.7 \times \lambda} \int_{2.7 \times \lambda}^{L / 2} \sigma_{\text {developed }} \mathrm{d} x
\end{gathered}
$$

Resulting average surface charge values normalized with theory at different $\lambda / H$ and $\mathrm{pH}$ values are plotted in Fig. 6 . Fig. 6(a) presents the normalized difference between the average surface charge on the corner and theory. Now, our objective was to find a suitable modeling approach for the behavior of each mechanism. The Sigmoid function family emerged as the best candidate as they are frequently used for the modeling of $\mathrm{pH}$ dependent behaviors in the literature widely. ${ }^{79}$ A logarithmic distribution, on the other hand, was found to be more appropriate for the corner effects.

The logarithmic function given in eqn (9) provides the best description for the behavior observed in the corner surface charging. Eqn (9) is composed of two second-order functions of pH while a logarithmic dependence appeared based on EDL thickness, as expected. The following functions presenting $\mathrm{pH}$ dependence are given in eqn (10). Results of eqn (9) and (10) are plotted in Fig. 6a (represented by solid lines) illustrating good agreement obtained with the numerical calculations. It should also be noted that eqn (9) became a general description for surface charging of any corner geometry as a function of $\mathrm{pH}$ and EDL thickness.

$$
\sigma_{\text {corner-ave }} / \sigma_{\text {theo }}=1+(f[\mathrm{pH}]+g[\mathrm{pH}] \times \ln (\lambda))
$$

where,

$$
\begin{gathered}
f[\mathrm{pH}]=-0.00923 \mathrm{pH}^{2}+0.12431 \mathrm{pH}-0.36763 \\
g[\mathrm{pH}]=0.00195 \mathrm{pH}^{2}-0.03113 \mathrm{pH}+0.11879
\end{gathered}
$$

A sigmoid function, presented in eqn (11), was found to describe the surface charge behavior of the nanochannel in both developing and developed regions best. This function consists of four different parameters and we formulized them based on the physics involved in the surface charging. First, we attempted to implement the overall overlap effect using a hyperbolic tangent function which gets zero value at low $\lambda / H$ values and converges to a constant as the $\lambda / H \rightarrow \infty$. The square of the hyperbolic tangent function for $p_{1}$ showed a good fit to the calculated surface charge values. The other two parameters, $p_{2}$ and $p_{3}$, were determined as the functions of $\lambda / H$ which describes $\mathrm{pH}$ dependence at different overlaps together with the final fourth parameter chosen directly as the $\mathrm{pH}$ of the solution.

$$
\sigma_{\text {developed }} / \sigma_{\text {theo }}=1-\left(p_{1}-\frac{p_{1}}{1+10^{\left(p_{2}-\mathrm{pH}\right) p_{3}}}\right)
$$

Eqn (11) was applied on average surface charge measured in both developing and developed regions given in Fig. $6 \mathrm{~b}$ and $\mathrm{c}$ (represented by solid lines). It should be noted here that the developing charge behavior represents the average of surface charges given in Fig. 5(e)-(g) where corner effects were removed. Clearly, the surface charge in the developing region after removing corner effects is equal to the developed part's surface charge at low overlaps since the ionic concentration in the channel is similar to the reservoir. As the overlap increases, the channel's ionic concentration differs from the reservoir and a so-called "second overlap" develops axially from the channel inlet and outlet such that the surface charge values become different from the developed region. However, this effect becomes almost negligible through the averaging such that the developing part surface charges are only slightly different from the developed
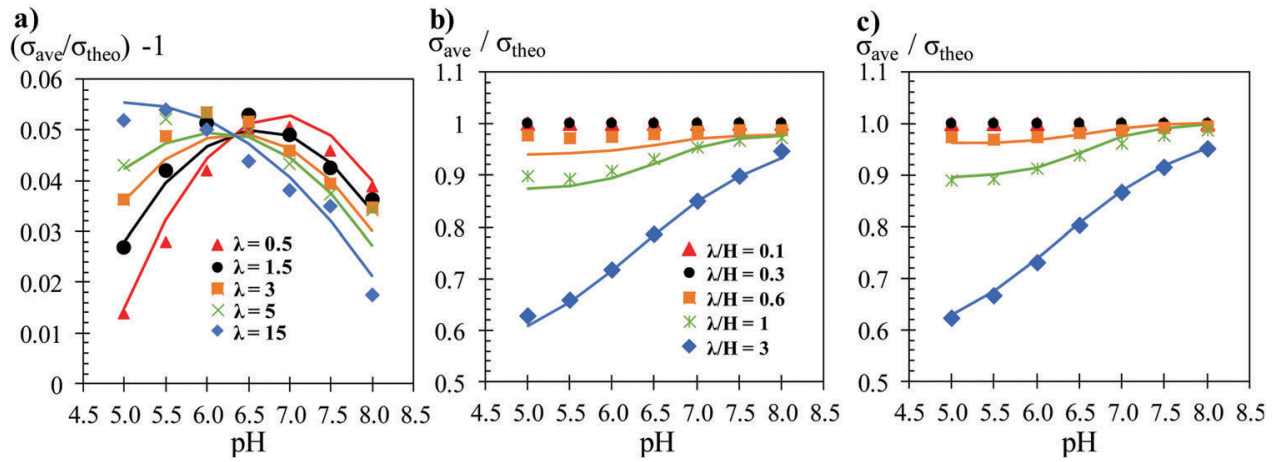

Fig. 6 Normalized average surface charge densities measured (a) on a corner through $0 \rightarrow 2.7 \times \lambda$, (b) in the developing region $(0 \rightarrow 2.7 \times \lambda)$ after removing corner effects, and (c) in the rest of the channel $(2.7 \times \lambda \rightarrow L)$. Markers represent numerical data and solid lines are the models. 
region values. For such a case, the parameter set given in eqn (12) recovered the behavior obtained in both electrokinetically developing and developed regions. Good agreements were obtained between the numerical results and models as seen in Fig. $6 \mathrm{~b}$ and c. The general s-shaped $\mathrm{pH}$ dependence of the surface charge density of silica is well described by our models.

$$
\begin{aligned}
& p_{1}=0.58 \tanh ^{2}\left(0.54 \frac{\lambda}{H}\right), \quad p_{2}=6.5307\left(\frac{\lambda}{H}\right)^{-0.034}, \\
& p_{3}=0.9806\left(\frac{\lambda}{H}\right)^{-0.519}
\end{aligned}
$$

The first additive is the inlet/outlet effect that appears as a function of $\lambda / L$ which will be effective for channel lengths comparable to the double layer thickness or else will have no contribution for negligible $\lambda / L$ values. The second part comes as a correction for double layer overlap as a function of $\lambda / H$. The asymptotic behavior comes with the hyperbolic tangent function begetting negligible values for negligible $\lambda / H$ cases and reaches a constant at high $\lambda / H$. For a channel whose length and height is much larger than the EDL thickness of the surrounding liquid, both the second and third terms in eqn (14) become negligible and the average surface charge of the channel remains the same as theoretical predictions.

$$
\sigma_{\text {channel,ave }}=\left(\begin{array}{c}
1+\frac{5.4 \lambda}{L}\left(\begin{array}{c}
\left(0.00195 \mathrm{pH}^{2}-0.03113 \mathrm{pH}+0.11879\right) \ln (\lambda) \\
-0.00923 \mathrm{pH}^{2}+0.12431 \mathrm{pH}-0.38763
\end{array}\right) \\
-\tanh ^{2}\left(0.54 \frac{\lambda}{H}\right)\left(\begin{array}{c}
0.58-\frac{0.58}{1+10}\left[6.5307\left(\frac{\lambda}{H}\right)^{-0.034}-\mathrm{pH}\right]\left[0.9806\left(\frac{\lambda}{H}\right)^{-0.519}\right]
\end{array}\right)
\end{array}\right) \sigma_{\text {theory }}
$$

As a last step, we defined an average surface charge for a nano-channel as given in eqn (13). Since the local surface charge variation in a developing region was found mostly due to the effect of corners, our equation became final in eqn (14) as the combination of corner surface charging and the developed surface charge of the corresponding channel.

$$
\begin{aligned}
\sigma_{\text {channel ave }}= & \left(\frac{2.7 \times \lambda}{L / 2}\right) \sigma_{\text {developing,ave }} \\
& +\left(\frac{L / 2-2.7 \times \lambda}{L / 2}\right) \sigma_{\text {developed }}
\end{aligned}
$$

Finally, we combined the above developed surface charge models given through eqn (9)-(12) into eqn (13). This final form of the model clearly shows the surface charging behavior of a nano-channel with a finite length and height. The two main mechanisms, inlet/oulet effect and overlap effect, are incorporated into general theoretically calculated surface charge as additions.

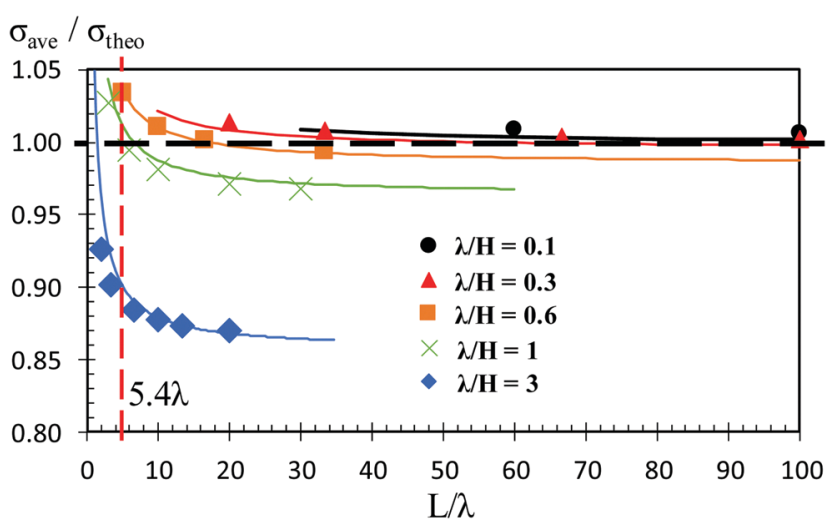

Fig. 7 Nanochannel average surface charge results (for $\mathrm{pH}=7$ ) from the extended theory (solid lines) given by eqn (14) in comparison with numerical calculations at different channel lengths.
This general equation calculates corresponding effects as an average inside certain length scales defined here. Thus, eqn (14) can be accurate for channels equal to or longer than $5.4 \times \lambda$. For such a case, we tested our proposed extended theory on different length nano-channels under different $\lambda / H$ conditions. In Fig. 7 , the channel average surface charges of different $L / H$ and $\lambda / H$ cases are given with the predictions of our proposed model. Eqn (14) gives very accurate results for high $L / \lambda$ at any $\lambda / H$ values. However, as the channel length becomes smaller than $5.4 \times \lambda$, the model starts to slightly diverge from numerical data, as expected.

Overall, for a channel whose length is much larger than the EDL thickness of the surrounding liquid $(L>50 \times \lambda)$, the electrokinetically developing region covers a negligible portion of the channel such that average surface charge becomes independent of channel length. For such a case, the nano-channel's surface charge becomes distinctly lower than the theoretical predictions if its height equals or is smaller than the EDL thickness $(\lambda / H \geq 1)$. However, as the channel length decreases, the contribution of the developing region increases and even dominates the average surface charge. Since the entrance effects create higher surface charge values than the developed section, decrease of channel length increases the nano-channel's average surface charge, which may reach and even exceed the theoretical value.

\section{Conclusions}

Surface charge density of silica nano-channels is characterized as a function of channel dimensions and solution conditions. In contrast to many studies that examine charging behavior of surfaces independent of the system dimensions, this study involves the effects of system size as well. Based on the results of numerical calculations, we found that surface charge density is not just a material property, but is dependent on the confinement 
size in addition to the solution conditions. In this regard, we calculated the local surface charge density based on the local ionic concentrations for different scenarios such as various channel length and solution parameters. This way, we evaluated both the local and average surface charge density of the nano channel and associated the effects of EDL overlap and inlet/outlet effects to the surface charging behavior of the silica surface. The results showed that EDL overlap, which is characterized by the ratio of EDL thickness to the channel height, significantly causes the surface charge density to deviate from theoretical calculations. The higher this ratio is, the less the surface charge density becomes on the surface. For the overlap ratios less than 0.3 , the theory can estimate the surface charge density except at the entrance and exit regions of the channel. For higher overlap ratios, the surface charge density may be as low as $60 \%$ of the theory (at $\lambda / H=3$ ) depending on the $\mathrm{pH}$. On the other hand, local surface charge values at the entrance regions of the nano channels were found to be almost twice the surface charge of developed sections. This behavior is attributed to both curvature effects at the inlet/outlet regions and new ionic equilibrium between the reservoir and channel in the axial direction. These entrance effects become dominant for shorter channels resulting in an increase in the average charge density such that the surface charge of the nano-channel can reach and even exceed theoretical estimations for channel lengths comparable with the EDL thickness $(\sim 3 \lambda)$. By using numerical results, a phenomenological model for the average surface charge density is formed, which includes the effects of EDL overlap and the electrokinetic development region as a function of channel height and length. The proposed model extends the existing theory as a function of EDL thickness, pH, channel length and channel height, successfully. The results are important for current nanomembrane applications with short pore lengths.

\section{Conflicts of interest}

There are no conflicts to declare.

\section{Acknowledgements}

The authors would like to thank Izmir Institute of Technology for the support under the Grant Number 2017-IYTE-49. The authors would also like to thank Center for Scientific Computation at Southern Methodist University.

\section{References}

1 J. J. Kasianowicz, E. Brandin, D. Branton and D. W. Deamer, Characterization of individual polynucleotide molecules using a membrane channel, Proc. Natl. Acad. Sci. U. S. A., 1996, 93, 13370-13773.

2 S. K. Min, W. Y. Kim, Y. Cho and K. S. Kim, Fast DNA sequencing with a graphene-based nanochannel device, Nat. Nanotechnol., 2011, 6, 162-165.
3 A. Meller, L. Nivon and D. Branton, Voltage-Driven DNA Translocations through a Nanopore, Phys. Rev. Lett., 2001, 86, 3435-3438.

4 M. N. V. Ravi Kumar, U. Bakowsky and C. Lehr, Preparation and characterization of cationic PLGA nanospheres as DNA carriers, Biomaterials, 2004, 25, 1771-1777.

5 H. Q. Mao, K. Roy, V. L. Troung-Le, K. A. Janes, K. Y. Lin, Y. Wang, J. T. August and K. W. Leong, Chitosan-DNA nanoparticles as gene carriers: synthesis, characterization and transfection efficiency, J. Controlled Release, 2001, 70, 399-421.

6 P. Calvo, C. Remunan-Lopez, J. Vila-Jato and M. Alonso, Chitosan and Chitosan/Ethylene Oxide-Propylene Oxide Block Copolymer Nanoparticles as Novel Carriers for Proteins and Vaccines, J. Appl. Polym. Sci., 1997, 63, 125-132.

7 C. Kneuer, M. Sameti, E. G. Haltner, T. Schiestel, H. Schirra, H. Schmidt and C. M. Lehr, Silica nanoparticles modified with aminosilanes as carriers for plasmid DNA, Int. J. Pharm., 2000, 196, 257-261.

8 K. Roy, H. Q. Mao, S. K. Huang and K. W. Leong, Oral gene delivery with chitosan-DNA nanoparticles generates immunologic protection in a murine model of peanut allergy, Nat. Med., 1999, 5, 387-391.

9 S. W. Kowalczyk, A. R. Hall and C. Dekker, Detection of Local Protein Structures along DNA Using Solid-State Nanopores, Nano Lett., 2009, 10, 324-328.

10 E. C. Yusko, J. M. Johnson, S. Majd, P. Prangkio, R. C. Rollings, J. Li, J. Yang and M. Mayer, Controlling protein translocation through nanopores with bio-inspired fluid walls, Nat. Nanotechnol., 2011, 6, 253-260.

11 A. Han, M. Creus, G. Schürmann, V. Linder, T. R. Ward, N. F. de Rooij and U. Staufer, Label-Free Detection of Single Protein Molecules and Protein-Protein Interactions Using Synthetic Nanopores, Anal. Chem., 2008, 80, 4651-4658.

12 M. Kaasalainen, E. Mäkilä, J. Riikonen, M. Kovalainen, K. Järvinen, K. H. Herzig, V. P. Lehto and J. Salonen, Effect of isotonic solutions and peptide adsorption on zeta potential of porous silicon nanoparticle drug delivery formulations, Int. J. Pharm., 2012, 431, 230-236.

13 W. J. Lan, D. A. Holden, J. Liu and H. S. White, PressureDriven Nanoparticle Transport across Glass Membranes Containing a Conical-Shaped Nanopor, J. Phys. Chem. C, 2011, 115, 18445-18452.

14 W. J. Lan, D. A. Holden, B. Zhang and H. S. White, Nanoparticle Transport in Conical-Shaped Nanopores, Anal. Chem., 2011, 83, 3840-3847.

15 C. Dekker, Solid-state nanopores, Nat. Nanotechnol., 2007, 2, 209-215.

16 S. Howorka, S. Cheley and H. Bayley, Sequence-specific detection of individual DNA strands using engineered nanopores, Nat. Biotechnol., 2001, 19, 636-639.

17 S. Howorka and Z. Siwy, Nanopore analytics: sensing of single molecules, Chem. Soc. Rev., 2009, 38, 2360-2384.

18 B. Neumcke, Ion Flux Across Lipid Bilayer Membranes With Charged Surfaces, Biophysik, 1970, 6, 231-240.

19 S. Behrens and D. Grier, The Charge Of Glass And Silica Surfaces, J. Chem. Phys., 2001, 115, 6716-6721. 
20 R. Probstein, Physicochemical Hydrodynamics, John Wiley \& Sons, Hoboken, 2005, pp. 161-200.

21 J. H. Masliyah and S. Bhattacharjee, Electrokinetic and colloid transport phenomena, John Wiley \& Sons, New Jersey, 2006.

22 T. Humplik, J. Lee, S. O'Hern, B. Fellman, M. Baig, S. Hassan, M. Atieh, F. Rahman, T. Laoui and R. Karnik, et al., Nanostructured Materials For Water Desalination, Nanotechnology, 2011, 22, 292001.

23 L. Fu, Analysis of electroosmotic flow with step change in zeta potential, J. Colloid Interface Sci., 2003, 258(2), 266-275.

24 M. Mao, J. D. Sherwood and S. Ghosal, Electro-osmotic flow through a nanopore, J. Fluid Mech., 2014, 749, 167-183.

25 N. Laohakunakorn and U. F. Keyser, Electroosmotic flow rectification in conical nanopores, Nanotechnology, 2015, 26(27), 275202.

26 M. Ali, B. Yameen, J. Cervera, P. Ramírez, R. Neumann, W. Ensinger, W. Knoll and O. Azzaroni, Layer-By-Layer Assembly of Polyelectrolytes Into Ionic Current Rectifying Solid-State Nanopores: Insights From Theory And Experiment, J. Am. Chem. Soc., 2010, 132, 8338-8348.

27 C. L. Sd Ren, Y. Hu, D. Li, C. Werner and A. New, Model for the Electrical Double Layer Interaction between Two Surfaces in Aqueous Solutions, J. Adhes., 2010, 80(9), 831-849.

28 M. Jia and T. Kim, Multiphysics simulation of ion concentration polarization induced by nanoporous membranes in dual channel devices, Anal. Chem., 2014, 86(15), 7360-7367.

29 L. Cao, F. Xiao, Y. Feng, W. Zhu, W. Geng, J. Yang, X. Zhang, N. Li, W. Guo and L. Jiang, Anomalous Channel-Length Dependence in Nanofluidic Osmotic Energy Conversion, Adv. Funct. Mater., 2017, 27(9), 1.

30 D. Jing and B. Bhushan, Electroviscous Effect On Fluid Drag In A Microchannel With Large Zeta Potential, Beilstein J. Nanotechnol., 2015, 6, 2207-2216.

31 B. W. Ninham and V. A. Parsegian, Electrostatic Potential between Surfaces Bearing Ionizable Groups in Ionic Equilibrium with Physiologic Saline Solution, J. Theor. Biol., 1971, 31(3), 405-428.

32 J. N. Israelachvili and G. E. Adams, Measurement of Forces between Two Mica Surfaces in Aqueous Electrolyte Solutions in the Range 0-100 Nm, J. Chem. Soc., Faraday Trans. 1, 1978, 74, 975.

33 R. M. Pashley and J. N. Israelachvili, Dlvo and Hydration Forces between Mica Surfaces in $\mathrm{Mg}^{2+}, \mathrm{Ca}^{2+}, \mathrm{Sr}^{2+}$, and $\mathrm{Ba}^{2+}$ chloride Solutions, J. Colloid Interface Sci., 1984, 97(2), 446-455.

34 V. E. Shubin and P. Kékicheff, Electrical Double Layer Structure Revisited via a Surface Force Apparatus: Mica Interfaces in Lithium Nitrate Solutions, J. Colloid Interface Sci., 1993, 155(1), 108-123.

35 J. P. Chapel, Electrolyte Species Dependent Hydration Forces between Silica Surfaces, Langmuir, 1994, 10(11), 4237-4243.

36 H. J. Butt, Measuring Electrostatic, van der Waals, and Hydration Forces in Electrolyte Solutions with an Atomic Force Microscope, Biophys. J., 1991, 60(6), 1438-1444.

37 P. Kékicheff and O. Spalla, Long-Range Electrostatic Attraction between Similar, Charge-Neutral Walls, Phys. Rev. Lett., 1995, 75(9), 1851-1854.
38 R. Pericet-Camara, G. Papastavrou and M. Borkovec, Effective Charge of Adsorbed Poly(amidoamine) Dendrimers from Direct Force Measurements, Macromolecules, 2009, 42(5), 1749-1758.

39 M. Dishon, O. Zohar and U. Sivan, From Repulsion to Attraction and back to Repulsion: The Effect of $\mathrm{NaCl}, \mathrm{KCl}$, and $\mathrm{CsCl}$ on the Force between Silica Surfaces in Aqueous Solution, Langmuir, 2009, 25(5), 2831-2836.

40 I. Popa, P. Sinha, M. Finessi, P. Maroni, G. Papastavrou and M. Borkovec, Importance of Charge Regulation in Attractive Double-Layer Forces between Dissimilar Surfaces, Phys. Rev. Lett., 2010, 104(22), 228301.

41 C. Hughes, L.-H. Yeh and S. Qian, Field Effect Modulation of Surface Charge Property and Electroosmotic Flow in a Nanochannel: Stern Layer Effect, J. Phys. Chem. C, 2013, 117(18), 9322-9331.

42 G. Trefalt, S. H. Behrens and M. Borkovec, Charge Regulation in the Electrical Double Layer: Ion Adsorption and Surface Interactions, Langmuir, 2016, 32(2), 380-400.

43 T. W. Healy, D. Chan and L. R. White, Colloidal behaviour of materials with ionizable group surfaces, Pure Appl. Chem., 1980, 52(5), 1207-1219.

44 G. M. Bell and G. C. Peterson, Forces between dissimilar colloidal plates for various surface conditions. II. Equilibrium adsorption effects, Can. J. Chem., 1981, 59(13), 1888-1897.

$45 \mathrm{~S}$. H. Behrens and M. Borkovec, Electrostatic Interaction of Colloidal Surfaces with Variable Charge, J. Phys. Chem. B, 1999, 103(15), 2918-2928.

46 M. Sadeghi, M. H. Saidi and A. Sadeghi, Electroosmotic flow and ionic conductance in a $\mathrm{pH}$-regulated rectangular nanochannel, Phys. Fluids, 2017, 29(6), 062002.

47 M. Sadeghi, M. H. Saidi, A. Moosavi and A. Sadeghi, Geometry effect on electrokinetic flow and ionic conductance in pH-regulated nanochannels, Phys. Fluids, 2017, 29(12), 122006.

48 A. Alizadeh, M. E. Warkiani and M. Wang, Manipulating electrokinetic conductance of nanofluidic channel by varying inlet pH of solution, Microfluid. Nanofluid., 2017, 21(3), 52.

49 K. Huang and R. Yang, Electrokinetic Behaviour Of Overlapped Electric Double Layers In Nanofluidic Channels, Nanotechnology, 2007, 18, 115701.

50 R. Nosrati, M. Hadigol, M. Raisee and A. Nourbakhsh, Numerical Modeling Of Electroosmotic Nanoflows With Overlapped Electric Double Layer, J. Comput. Theor. Nanosci., 2012, 9, 2228-2239.

51 A. Golovnev and S. Trimper, Steady State Solution Of The Poisson-Nernst-Planck Equations, Phys. Lett. A, 2010, 374, 2886-2889.

52 J. Holt, Fast Mass Transport Through Sub-2-Nanometer Carbon Nanotubes, Science, 2006, 312, 1034-1037.

53 H. Verweij, M. Schillo and J. Li, Fast Mass Transport Through Carbon Nanotube Membranes, Small, 2007, 3, 1996-2004.

54 L. Cheng and L. Guo, Entrance Effect On Ion Transport In Nanochannels, Microfluid. Nanofluid., 2010, 9, 1033-1039.

55 R. Yang, T. Tseng and C. Chang, End Effects On ElectroOsmotic Flows In Micro-Channels, J. Micromech. Microeng., 2004, 15, 254-262. 
56 J. Berry, M. Davidson and D. Harvie, Electrokinetic Development Length Of Electroviscous Flow Through A Contraction, ANZIAM Journal, 2011, 52, 837.

57 Y. Ai, M. Zhang, S. W. Joo, M. A. Cheney and S. Qian, Effects of Electroosmotic Flow on Ionic Current Rectification in Conical Nanopores, J. Phys. Chem. C, 2010, 114(9), 3883-3890.

58 F. Baldessari and J. G. Santiago, Electrokinetics in nanochannels: part I. Electric double layer overlap and channel-towell equilibrium, J. Colloid Interface Sci., 2008, 325(2), 526-538.

59 A. Popat, S. B. Hartono, F. Stahr, J. Liu, S. Z. Qiao and G. Q. M. Lu, Mesoporous silica nanoparticles for bioadsorption, enzyme immobilisation, and delivery carriers, Nanoscale, 2011, 3(7), 2801-2818.

60 X. Yang, Z. Shen, B. Zhang, J. Yang, W. X. Hong, Z. Zhuang and J. Liu, Silica nanoparticles capture atmospheric lead: Implications in the treatment of environmental heavy metal pollution, Chemosphere, 2013, 90, 653-659.

61 M. Barisik, S. Atalay, A. Beskok and S. Qian, Size Dependent Surface Charge Properties Of Silica Nanoparticles, J. Phys. Chem. C, 2014, 118, 1836-1842.

62 G. Karniadakis, A. Beskök and N. Aluru, Microflows And Nanoflows, Springer-Verlag, New York, New York, 1st edn, 2005.

63 D. Li, Electrokinetics In Microfluidics, Elsevier/Academic Press, Amsterdam [u.a.], 2004.

64 R. Hunter, Zeta Potential In Colloid Science, Academic Press, London, 1981.

65 S. Qian and Y. Ai, Electrokinetic Particle Transport In Micro-/ Nanofluidics, CRC Press, Boca Raton, 2012.

66 M. Polat and H. Polat, Analytical Solution Of PoissonBoltzmann Equation For Interacting Plates Of Arbitrary Potentials And Same Sign, J. Colloid Interface Sci., 2010, 341, 178-185.

67 D. McCormack, S. L. Carnie and D. Y. C. Chan, Calculations of Electric Double-Layer Force and Interaction Free Energy between Dissimilar Surfaces, J. Colloid Interface Sci., 1995, 169(1), 177-196.
68 W. D. Murphy, J. A. Manzanares, S. Mafe and H. Reiss, A numerical study of the equilibrium and nonequilibrium diffuse double layer in electrochemical cells, J. Phys. Chem., 1992, 96(24), 9983-9991.

69 B. J. Kirby, Micro-Nanoscale Fluid Mechanics Transport in Microfluidic Devices, Cornell University, New York, 2010.

70 D. Stein, M. Kruithof and C. Dekker, Surface-charge-governed ion transport in nanofluidic channels, Phys. Rev. Lett., 2004, 93(3), 035901.

71 H. Daiguji, P. Yang, A. J. Szeri and A. Majumdar, Electrochemomechanical Energy Conversion in Nanofluidic Channels, Nano Lett., 2004, 4(12), 2315-2321.

72 F. Tessier and G. W. Slater, Effective Debye length in closed nanoscopic systems: a competition between two length scales, Electrophoresis, 2006, 27(3), 686-693.

73 R. J. Yang, L. M. Fu and C. C. Hwang, Electroosmotic Entry Flow in a Microchannel, J. Colloid Interface Sci., 2001, 244(1), 173-179.

74 S. Bhattacharyya, Z. Zheng and A. T. Conlisk, Electro-osmotic flow in two-dimensional charged micro- and nanochannels, J. Fluid Mech., 2005, 540, 247-267.

75 S. Atalay, M. Barisik, A. Beskok and S. Qian, Surface Charge Of A Nanoparticle Interacting With A Flat Substrate, J. Phys. Chem. C, 2014, 118, 10927-10935.

76 J. Huang, B. G. Sumpter and V. Meunier, A universal model for nanoporous carbon supercapacitors applicable to diverse pore regimes, carbon materials, and electrolytes, Chemistry, 2008, 14(22), 6614-6626.

77 J. Huang, B. G. Sumpter and V. Meunier, Theoretical model for nanoporous carbon supercapacitors, Angew. Chem., Int. Ed., 2008, 47(3), 520-524.

78 J. Huang, R. Qiao, B. G. Sumpter and V. Meunier, Effect of diffuse layer and pore shapes in mesoporous carbon supercapacitors, J. Mater. Res., 2011, 25(08), 1469-1475.

79 W. Lambert and D. Middleton, Ph Hysteresis Effect With Silica Capillaries In Capillary Zone Electrophoresis, Anal. Chem., 1990, 62, 1585-1587. 